\title{
COMET TAXONOMY AND EVOLUTION
}

\author{
DAVID G. SCHLEICHER \\ Lowell Observatory \\ 1400 W. Mars Hill Rd. \\ Flagstaff, $A Z$ 86001, USA \\ E-mail dschleicher@lowell.edu
}

\begin{abstract}
Measurements of physical and dynamical properties of comets have yielded considerable evidence for variations in the origin and evolution of these primordial objects. Given these findings, one might reasonably expect to observe differences in the chemical composition as well. This paper reviews our current state of knowledge of the similarities and differences in the composition of comets, and summarizes the recent findings by A'Hearn et al. (1994) for two taxonomic classes of comets and evidence of compositional variation due to origin and/or evolution.
\end{abstract}

\section{Introduction}

Comets are widely believed to be the most pristine remnants from the epoch of solar system formation and, as such, can provide important clues to the physical and chemical processes which took place at that time. For this reason, much of cometary research is aimed at obtaining an understanding of the origin, basic compositional and physical characteristics, and fate of cometary nuclei. However, the acquisition of the first direct samples from a nucleus still lies in the future, and so compositional studies have necessarily relied on measurements of cometary comae constituents. While the in situ measurements of Comet $\mathrm{P} / \mathrm{Halley}$ by five spacecraft in 1986 (and subsequently of Comet P/Grigg-Skjellerup by Giotto) were extremely important in determining spatial densities within the coma and detecting species not observed from Earth, they were unable to address the issue of whether or not Halley's composition is "normal" and, therefore, typical of other comets.

Numerous papers in recent years have explored the dynamical evolution of cometary orbits and the evidence for two reservoirs of comets - the Oort cloud and the Kuiper belt - which are presumably the primary sources of dynamically new comets and Jupiter-family comets, respectively (cf. Duncan, Quinn, and Tremaine 1988; Mumma, Weissman, and Stern 1993). If this scenario is true, then compositional differences might be expected because the comets in these two groups would have formed at different locations and, thus, under different conditions within the proto-solar nebula. While not wholly immune from evolutionary processes while "stored" in either the Oort Cloud or Kuiper Belt (cf. Donn 1976; Stern 1990; Mumma, Weissman, and Sterns 1993), one would expect that any compositional differences would still be evident today. Thus far, quantitative studies of the chemical composition of comets - based on abundances and production rates of species within cometary comae - show only limited evidence for this type of trend with orbital dynamics.

Several spectroscopic studies prior to the 1980 s reached the conclusion that the general composition of all comets is nearly the same, based on the recurrence of 
many atomic and molecular features in cometary spectra, and sometimes crude quantitative measures of relative abundances (cf. Arpigny 1976). More recent photometric and spectrophotometric studies of the UV, visible, and near-IR portions of the spectrum (see Figure 1 for a representative composite spectrum of Comet $\mathrm{P} /$ Tuttle) have reached the same basic conclusion, based on computed production rates of the observed species (cf. A'Hearn and Millis 1980; Weaver et al. 1981; Newburn and Spinrad 1984, 1989; Cochran 1990; Cochran et al. 1992; A'Hearn et al. 1994). The other general conclusion of these studies has been that the gas-to-dust ratio of comets is not constant, but instead varies by over two orders of magnitude, with no obvious trend with age.

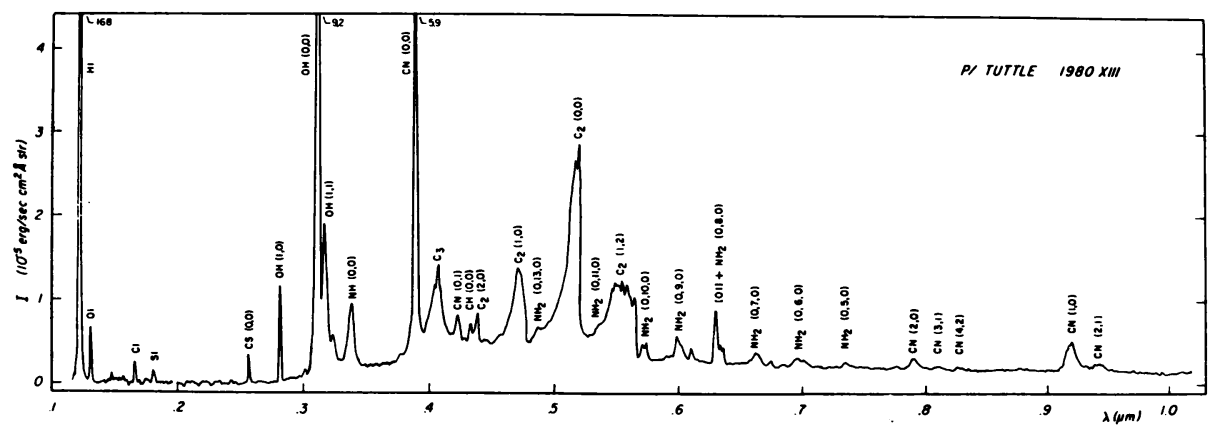

Fig. 1. Composite spectrum of Comet P/Tuttle (1980 XIII) is representative of the features observed in most cometary spectra. The UV portion of the spectrum was obtained with the IUE satellite (Weaver et al. 1981), while the remainder was obtained in two parts with the $1.54 \mathrm{~m}$ Catalina Station telescope of the University of Arizona. The composite was created by S. Larson and J. Johnson. Courtesy of S. Larson.

Radio observations, primarily of the $\mathrm{OH}$ molecule, have been obtained for many dozens of comets, but these have usually not been obtained concurrently with measurements of other species and, therefore, are not suitable for directly determining abundance ratios. In the last few years, observations of comets in the microwave region have become common, and have resulted in many new species being detected. The status of this new field and lists of the many species observed are given in several recent reviews (Krankowsky 1991; Mumma, Weissman, and Stern 1993; Crovisier 1992, 1994).

While it has been determined that the large majority of comets studied have the same basic composition, significant depletions or enhancements of certain species have also been measured in many comets. Evidence for abundance variations has been observed for several molecular species - in some cases apparently associated with the dust abundance - for which the number of comets measured is yet too small for meaningful statistical analyses to be performed. Examples include CO (Feldman 1983,1986 ) and $\mathrm{S}_{2}$ (Budzien and Feldman 1992) in the UV, $\mathrm{H}_{2} \mathrm{CO}$ (formaldehyde) (Colom et al. 1992; Mumma, Weissman, and Stern 1993) and $\mathrm{CH}_{3} \mathrm{OH}$ (methanol) 
(Hoban et al. 1991; Mumma et al. 1993) in the IR and microwave, and $\mathrm{N}_{2}{ }^{+}$(Lutz et al. 1993) in the visible. To investigate groupings and correlations of cometary composition, the remaining sections of this paper will necessarily concentrate on the findings based on the few larger sized data bases currently available.

\section{Data Bases for Compositional Studies of Comets}

To date, four long-term observational programs have obtained compositional measurements in a systematic manner for more than 15-20 comets. The earliest of these was begun in 1976 by A'Hearn and Millis (1980). Narrowband filter photometry has now been obtained by this group for a total of 88 comets. A'Hearn et al. (1994) report on summary results for the 85 comets observed through 1992, along with the outcome from taxonomic analyses performed on a subset of 41 comets for which multiple observations of $\mathrm{OH}, \mathrm{NH}, \mathrm{CN}, \mathrm{C}_{2}$, and $\mathrm{C}_{3}$ were obtained. Their taxonomic results will be summarized in Section 3.

Two programs of visible spectrophotometry have been undertaken. The ongoing McDonald Observatory Faint Comet Survey by Cochran (1987) and colleagues has observed approximately 100 comets since its inception in 1979. Cochran, Green, and Barker (1989) analyzed 29 "low activity" comets, and Cochran (1990) briefly summarized results for the $\mathrm{CN}, \mathrm{C}_{2}$, and $\mathrm{C}_{3}$ abundances of a larger sample of 48 comets. These studies concluded that only one comet - P/Giacobini-Zinner - stood out as having substantially different relative abundances; however, many of the comets have only "uninteresting" upper limits for $\mathrm{C}_{2}$ and $\mathrm{C}_{3}$. Giacobini-Zinner was, in fact, identified in several studies as having $\mathrm{C}_{2}$ and $\mathrm{C}_{3}$ depleted by more than a factor of 5 and $\mathrm{NH}$ and $\mathrm{NH}_{2}$ depleted by lesser amounts (cf. Cochran and Barker 1987; Schleicher, Millis, and Birch 1987; Beaver et al. 1990). Recently, detailed analyses from the McDonald survey were extended to $\mathrm{CH}$ and $\mathrm{NH}_{2}$ for 17 comets (Cochran et al. 1992), with none displaying unusual abundances.

The other program of visible spectrophotometry, begun in 1980 by Newburn and Spinrad, measures the emission of $\mathrm{CN}, \mathrm{C}_{2}, \mathrm{C}_{3}, \mathrm{CH}$, and $\mathrm{O}\left[{ }^{1} \mathrm{D}\right]$. In their initial paper which included data for 17 comets, Newburn and Spinrad (1984) identified 2 comets - P/Borrelly and P/Swift-Gehrels - as being depleted in $\mathrm{C}_{2}$ and $\mathrm{C}_{3}$. In their more recent paper for 25 comets (including the original 17), Newburn and Spinrad (1989) note a clear trend of the $\mathrm{C}_{2}$-to-CN ratio with heliocentric distance. However, Cochran et al. (1992) do not see a variation with distance, implying that any such trend may be an artifact of the scalelengths chosen for the reduction from column density to production rate. Newburn and Spinrad (1989) do see a clear correlation of $\mathrm{CN}$ abundance with dust, strongly implying a dust source for $\mathrm{CN}$, in addition to the generally assumed parent gas source. This correlation is demonstrated in Figure 2. A similar effect is also seen by A'Hearn et al. (1994) for several individual comets which exhibit abundance ratio variations with heliocentric distance.

It was already noted in Section 1 that the gas-to-dust ratio varies enormously among comets. In their analysis of dust for 18 comets, Storrs, Cochran, and Barker (1992) show evidence that the heliocentric distance dependence of Afo, a measure of dust production, is less steep than is the dependence of the production rate of CN, a conclusion supported by A'Hearn et al. (1994). Storrs, Cochran, and Barker 


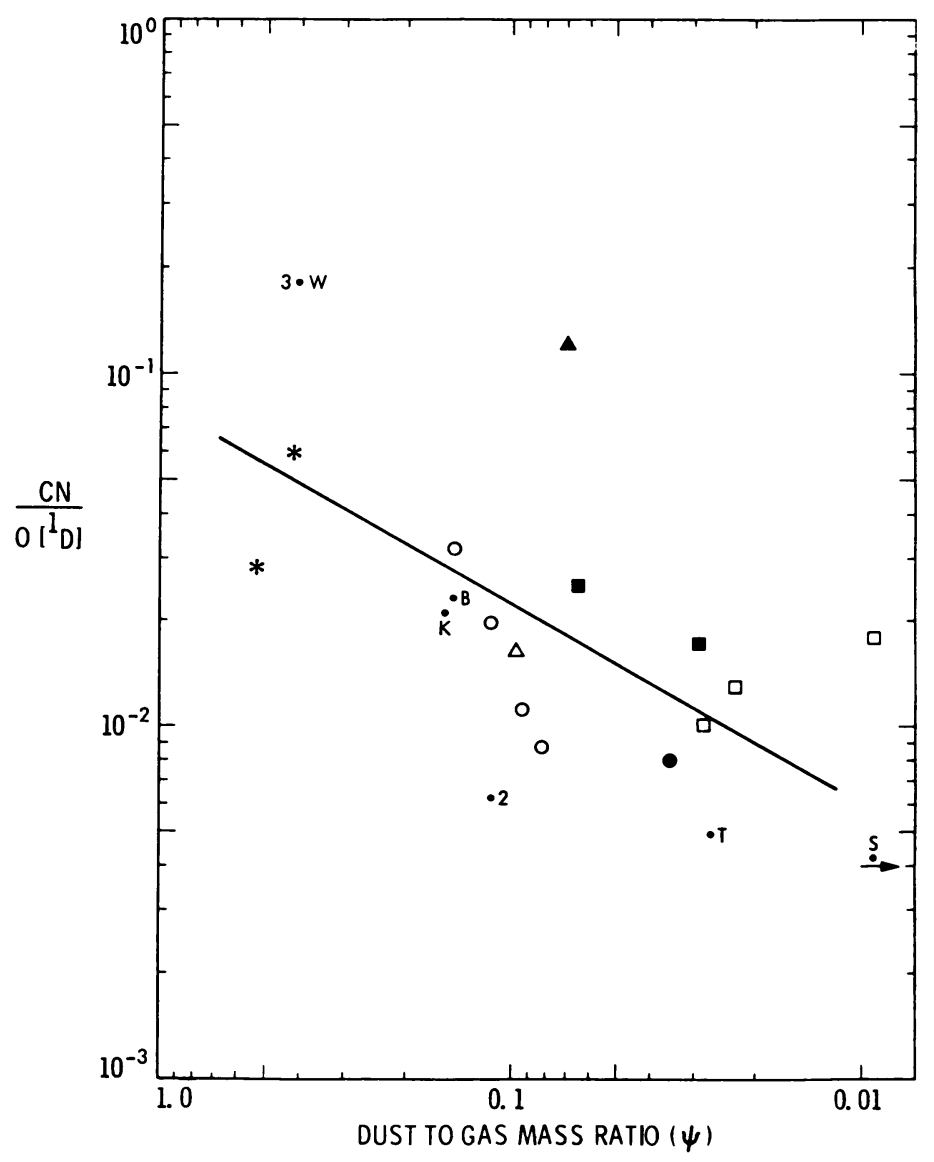

Fig. 2. The correlation of $\mathrm{CN}$ to dust is evident in this plot of the CN-to-O $\left[{ }^{1} \mathrm{D}\right]$ ratio with respect to the dust-to-gas ratio. See the original paper for the correspondence between symbols and comets. From Newburn and Spinrad (1989).

also indicate that the gas-to-dust ratio shows a trend - with the ratio increasing with increased gas $(\mathrm{CN})$ production - and attribute this effect to gas production through a porous mantle. While their assumption that $\mathrm{CN}$ is a good measure of the total gas production is true to first order, $\mathrm{A}^{\prime} \mathrm{Hearn}$ et al. show that the $\mathrm{CN}$-to-OH ratio varies by as much as a factor of 4 among comets, implying that further work is required to better understand the relationship of gas with dust grains.

Finally, the ultraviolet program by Feldman, A'Hearn, Festou, Weaver and collaborators makes use of the International Ultraviolet Explorer (IUE) satellite. Approximately 50 comets have been observed since 1978 . While the $\mathrm{OH}$ emission bands are ubiquitous in IUE spectra, other emission features are usually only observed in brighter comets. In more than a dozen comets for which CS was detected 
and measured, only $\mathrm{P} / \mathrm{Swift}-\mathrm{Tuttle}$ shows a significant variation from the mean CS-to-OH ratio, having a CS abundance that is high by more than a factor of two (Feldman, private communication).

\section{A Comet Taxonomy}

Until the last decade, the creation of a taxonomic classification system for comets based on chemical composition was stymied because too few species had been measured in too few comets. While the three more recent spectrophotometric studies discussed in Section 2 contain statistically useful numbers of comets, the results from these studies indicated that nearly all comets for which the usual molecular species were detected have about the same composition, and only a few comets clearly have significant anomalies. However, for many other comets only upper limits have been obtained for certain species due to the intrinsic faintness of the comets and/or the large amount of continuum in the spectrum.

An advantage of the narrowband filter photometry study by A'Hearn et al. (1994) is that relatively large photometer entrance apertures - usually between 30 and 150 arcsec - were employed in making the observations. The use of large apertures helps in several ways. For example, the effects of inaccurate scalelengths in the calculation of production rates are reduced because a much greater fraction of the comet is sampled. Moreover, this approach gives greater contrast of the emission bands to the underlying continuum resulting in many more actual detections of individual species. Another aspect of the narrowband filter photometry study by A'Hearn et al. is the simultaneous acquisition of $\mathrm{OH}$ measurements in the near-UV with measurements of the major emission features in the visible : $\mathrm{CN}, \mathrm{NH}, \mathrm{C}_{2}$, and $\mathrm{C}_{3}$. While both $\mathrm{OH}$ and $\mathrm{O}$ both are created by the dissociation of water, the bulk constituent of comets, deduction of the water production rate from $\mathrm{OH}$ is less dependent on imprecisely known parameters such as the branching ratio of $\mathrm{H}_{2} \mathrm{O}$, than are results based on measurements of $O\left[{ }^{1} \mathrm{D}\right]$. These aspects of the A'Hearn et al. program assisted in making taxonomic analyses viable, and it is primarily their results which are presented next.

To minimize the effects of spurious data and possible model dependencies in the computation of production rates, the A'Hearn et al. (1994) taxonomic analyses were restricted to a subset of the total data base of 85 comets. In particular, only data sets were included for which measurements of all 5 gas species $\left(\mathrm{OH}, \mathrm{CN}, \mathrm{C}_{2}\right.$, $\mathrm{C}_{3}$, and $\mathrm{NH}$ ) were made, and for which the comet's heliocentric distance was less than $3 \mathrm{AU}$. In addition, each included comet was required to have been observed on at least 2 nights and to have 3 or more individual data sets, as a verification of the quality of the data. These restrictions resulted in a subset consisting of 41 comets for which production rate ratios (i.e., abundance ratios) with respect to $\mathrm{OH}$ were determined. Log values of the mean ratios, weighted by the quality of the data, were computed for each comet, and the resulting 41 ratios for each minor species form the actual taxonomic database.

The most dramatic result for this database is shown in Figure 3, where the $\mathrm{C}_{2}$ to- $\mathrm{OH}$ production rate ratio is plotted as a function of the $\mathrm{CN}$-to- $\mathrm{OH}$ ratio. It is apparent that approximately $70 \%$ of the comets have a direct relationship of 
$\mathrm{C}_{2}$ with $\mathrm{CN}$, while the remaining comets have lower $\mathrm{C}_{2}$ abundances by varying amounts. This clear trend for the majority of comets indicates that both $\mathrm{CN}$ and $\mathrm{C}_{2}$ vary together, but that they both vary by as much as a factor of 3-4 with respect to $\mathrm{OH}$. Note that the line plotted in Figure 3 is not fitted to the data, but rather is a line with a slope of unity. This group is identified by A'Hearn et al. as having "typical" abundances. They also show that $\mathrm{C}_{3}$ follows the same relationship as $\mathrm{C}_{2}$, but with greater scatter, presumably due to poorer signal-to-noise ratio of the $\mathrm{C}_{3}$ data. In contrast, no obvious relation is seen when the $\mathrm{NH}-$ to- $\mathrm{OH}$ ratio is plotted versus the $\mathrm{CN}$-to- $\mathrm{OH}$ ratio. This indicates that for the "typical" class of comets the $\mathrm{CN}, \mathrm{C}_{2}$, and $\mathrm{C}_{3}$ are all related to each other much more strongly than to either $\mathrm{OH}$ or $\mathrm{NH}$. The remaining $30 \%$ of the comets form the second taxonomic group and exhibit significant depletions in the carbon-chain species $\left(\mathrm{C}_{2}\right.$ and $\left.\mathrm{C}_{3}\right)-$ by as much as a factor of 20 from the mean value for the typical group - while CN and NH both have "typical" abundances with respect to $\mathrm{OH}$.

As is evident from Figure 3, a transition region exists within which the appropriate classification is not obvious. Because of the direct relationship seen for $\mathrm{C}_{2}$ with $\mathrm{CN}$ for the majority of comets, along with the results from a principle component analysis (discussed later), A'Hearn et al. use the $\mathrm{C}_{2}$-to-CN ratio as the discriminator for classification. The resulting distributions of abundance ratios is shown in Figure 4, where histograms of each species with respect to both $\mathrm{OH}$ and CN are shown, and the "typical" comets are highlighted. Near-gaussian distributions are seen for the "typical" class in each case, while a long "tail" is seen for the carbon-chain species in the depleted class. Although the NH abundance is lower than average for several of the carbon-chain depleted comets (including previously mentioned Comet Giacobini-Zinner), it is not evident from these plots that additional classifications based on $\mathrm{NH}$ abundance are warranted. However, it it possible the groupings based on $\mathrm{NH}$ do exist within the apparently broad distribution, but are simply washed out due to the relatively poor $\mathrm{S} / \mathrm{N}$ of the $\mathrm{NH}$ measurements and the resulting larger associated errors. Finally, it should be noted that some comets were observed to change their location on the diagram (Figure 3) as a function of heliocentric distance and/or time. For instance, several "typical" comets move along the diagonal line designating this class in the course of their respective apparitions. While this behavior can be partially explained as an artifact of the scalelengths used in the Haser model for calculating production rates, A'Hearn et al. indicate that the majority of the effect is real. This implies the existence of inhomogeneities within some individual nuclei which match the chemical variations measured among different comets within the "typical" class. That is to say, the same highly correlated variation in the abundances of the three carbonbearing species with respect to water which is measured from one "typical" comet to another is also observed within some individual comets.

A'Hearn et al. also applied both the techniques of principle component analysis and cluster analysis to their taxonomic database. Because the database already consisted of four gas abundance ratios for each comet, the principle component analysis was not intended to reduce the number of variables to a smaller, physically meaningful parameter set. This contrasts to the situation in asteroid photometry where one can employ principle component analysis to reduce a large number of 


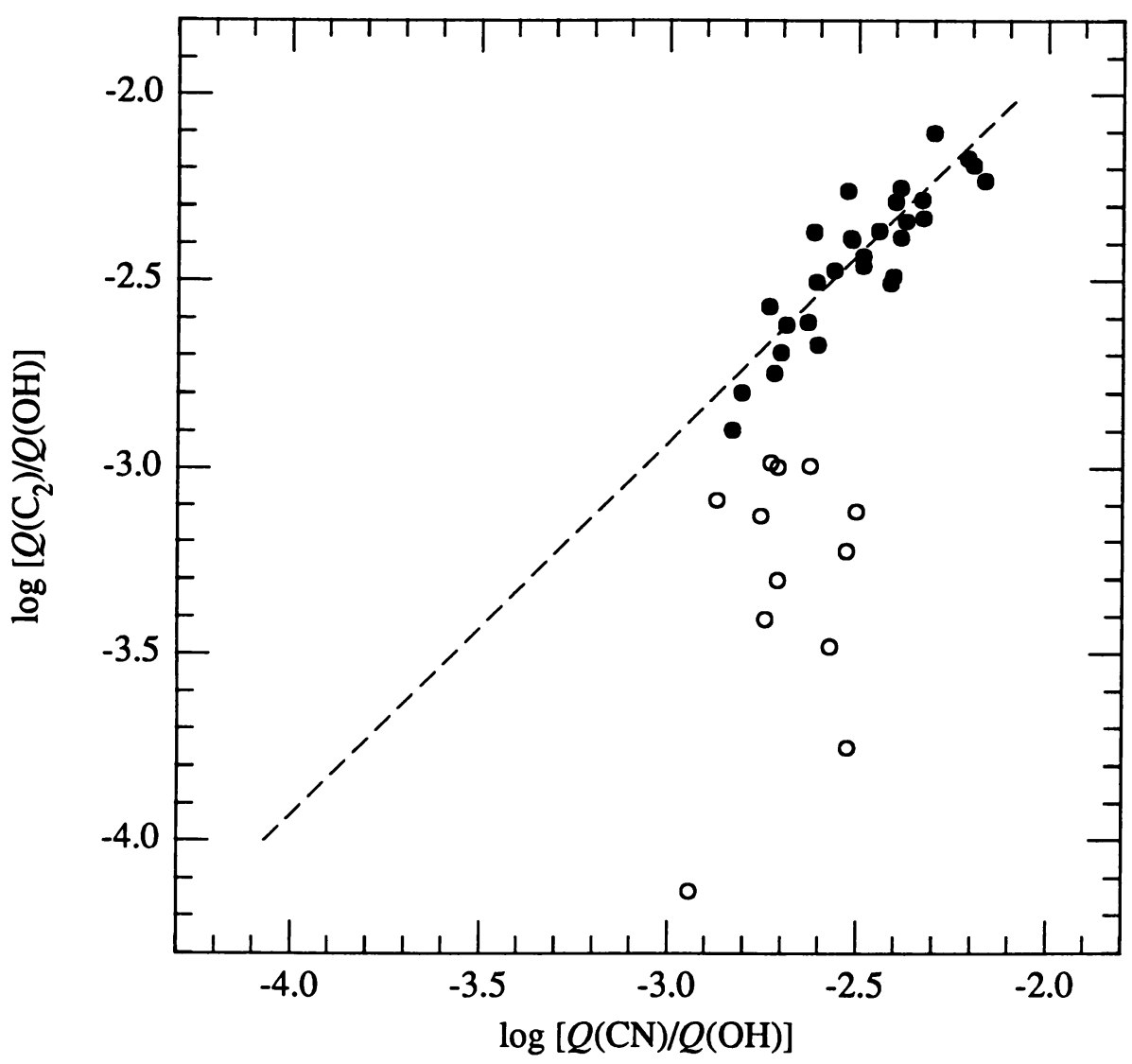

Fig. 3. Two taxonomic groups are identified based on the relative production rates of $\mathrm{OH}, \mathrm{CN}$, and $\mathrm{C}_{2}$ for the 41 comets contained within the $\mathrm{A}^{\prime} \mathrm{Hearn}$ et al. (1994) restricted database. Within the "typical" group (filled symbols), $\mathrm{CN}$ and $\mathrm{C}_{2}$ vary together with respect to $\mathrm{OH}$ (the line has a slope of unity). Based on $A^{\prime}$ Hearn et al. (1994).

filter magnitudes to a few diagnostic components (cf. Tholen 1984). In spite of this difference in situations, the analysis of the comet database results in the first principle component containing over $70 \%$ of the total variance, while the second principle component contains only about $15 \%$ of the variance. Not unexpectedly, the first principle component is primarily a near-equal combination of the $\mathrm{C}_{2}-$ to- $\mathrm{OH}$ and $\mathrm{C}_{3}-$ to- $\mathrm{OH}$ ratios, along with a smaller contribution from $\mathrm{CN}$-to- $\mathrm{OH}$. In other words, this axis goes from approximately the bottom and left-most data points shown in Figure 3 to the top-right points. The second principle component is nearly entirely composed of the $\mathrm{NH}-$ to- $\mathrm{OH}$ ratio, reflecting the generally broad distribution of this variable as seen in Figure 4. These results are therefore consistent with the basic taxonomic classifications of A'Hearn et al. 

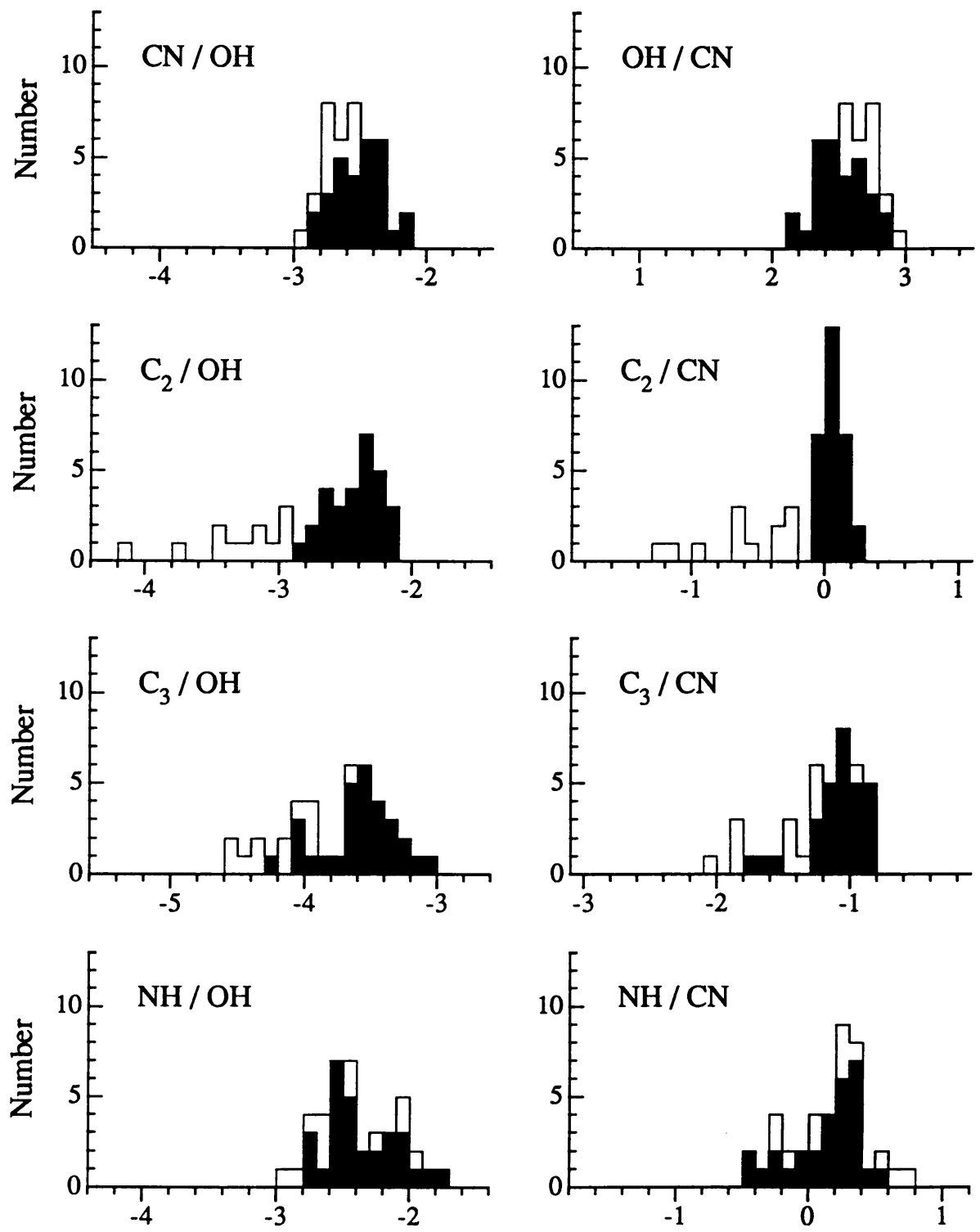

log Production Rate Ratio

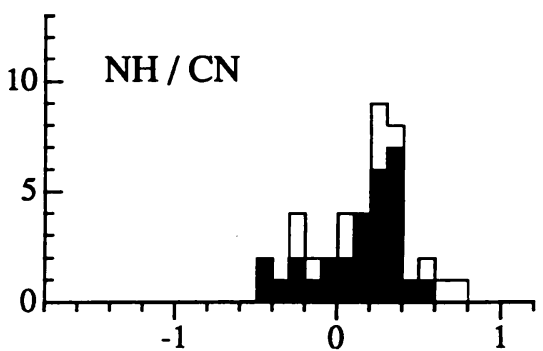

$\log$ Production Rate Ratio

Fig. 4. Histograms showing the distribution of production rate ratios for the restricted database of 41 comets. Shaded regions are comets which are part of the "typical" group, and the histograms have been shifted horizontally to align the mean values of the "typical" group. From A'Hearn et al. (1994). 
Using a complete linkage hierarchical clustering algorithm, A'Hearn et al. obtain two nearly equal-sized clusters or groups from their taxonomic database. These groups are basically the upper-half and lower-half of the points shown in Figure 3. The only significant difference between this cluster analysis grouping and the taxonomic groups identified previously is the placement of the comets at the lowerleft end of the region defining the "typical" group in the diagram. As already noted, A'Hearn et al. consider the strong correlation of the abundances of $\mathrm{CN}, \mathrm{C}_{2}$, and $\mathrm{C}_{3}$ for the majority of comets (i.e., comets along the diagonal line in Figure 3) as highly physically significant and, therefore, should override the cluster analysis result, since the clustering algorithm ignores this distinctive aspect of the data.

Comet Giacobini-Zinner was identified in Section 2 as a well-observed comet having depletions of several species. It is appropriate to consider Giacobini-Zinner as the prototype of A'Hearn et al.'s class of carbon-chain depleted comets, since it was identified as being unusual in its composition more than 60 years ago (Bobrovnikoff 1927). The most extreme example of this group from the A'Hearn et al. database is Comet P/Wolf-Harrington. Analysis of photometric and spectrophotometric data for this comet by Schleicher, Bus, and Osip (1993) indicate that both $\mathrm{C}_{2}$ and $\mathrm{C}_{3}$ are depleted by more than an order of magnitude with respect both to $\mathrm{OH}$ and to $\mathrm{CN}$. This extreme example of a carbon-chain depleted comet is shown in Figure 5. Even more unusual abundances were reported for Comet Yanaka (1988r) by Fink (1992) based on spectrophotometric measurements. However, unlike comets in the carbon-chain depleted group identified by A'Hearn et al., both $\mathrm{CN}$ and $\mathrm{C}_{2}$ were undetected in the Yanaka spectrum, while both $\mathrm{NH}_{2}$ and $\mathrm{O}\left[{ }^{1} \mathrm{D}\right]$ were present ( $\mathrm{OH}$ and $\mathrm{NH}$ were outside the spectral region and not observed). Based on a water production rate computed using the $\mathrm{O}\left[{ }^{1} \mathrm{D}\right]$ measurement and upper limits for $\mathrm{CN}$ and $\mathrm{C}_{2}$, Fink concludes that these species were depleted by a factor of at least 25 and 100, respectively, but that the $\mathrm{NH}_{2}$ has normal abundance. It is interesting to note that comparable depletions of $\mathrm{CN}$ and $\mathrm{C}_{2}$ with respect to water would place Yanaka near the lower-left corner of the A'Hearn et al. taxonomic diagram (Figure 3), close to where the diagonal line defining their "typical" group of comets extends.

\section{Compositional Evidence for Comet Evolution}

Much physical evidence exists indicating that comets evolve or age with time. For instance, dynamically new comets are observed to be preferentially brighter prior to perihelion than after, possibly due to the higher volatility of the original surface compared with material deeper within the nucleus (Whipple 1977). Well-established cases of short-period comets fading with successive apparitions and eventually disappearing are documented in the literature, while other comets have been mis-identified as asteroids due to their asteroidal appearance on some occasions (cf. Kresák 1991). However, changes in observing techniques, selection effects, sporadic variability, and changes induced by orbital perturbations must all be accounted for when interpreting a comet's behavior as evidence for evolution (Kresák, this volume; Rickman et al. 1991). Other expected evolutionary effects are often assumed, but data do not exist as yet to confirm them. For example, 

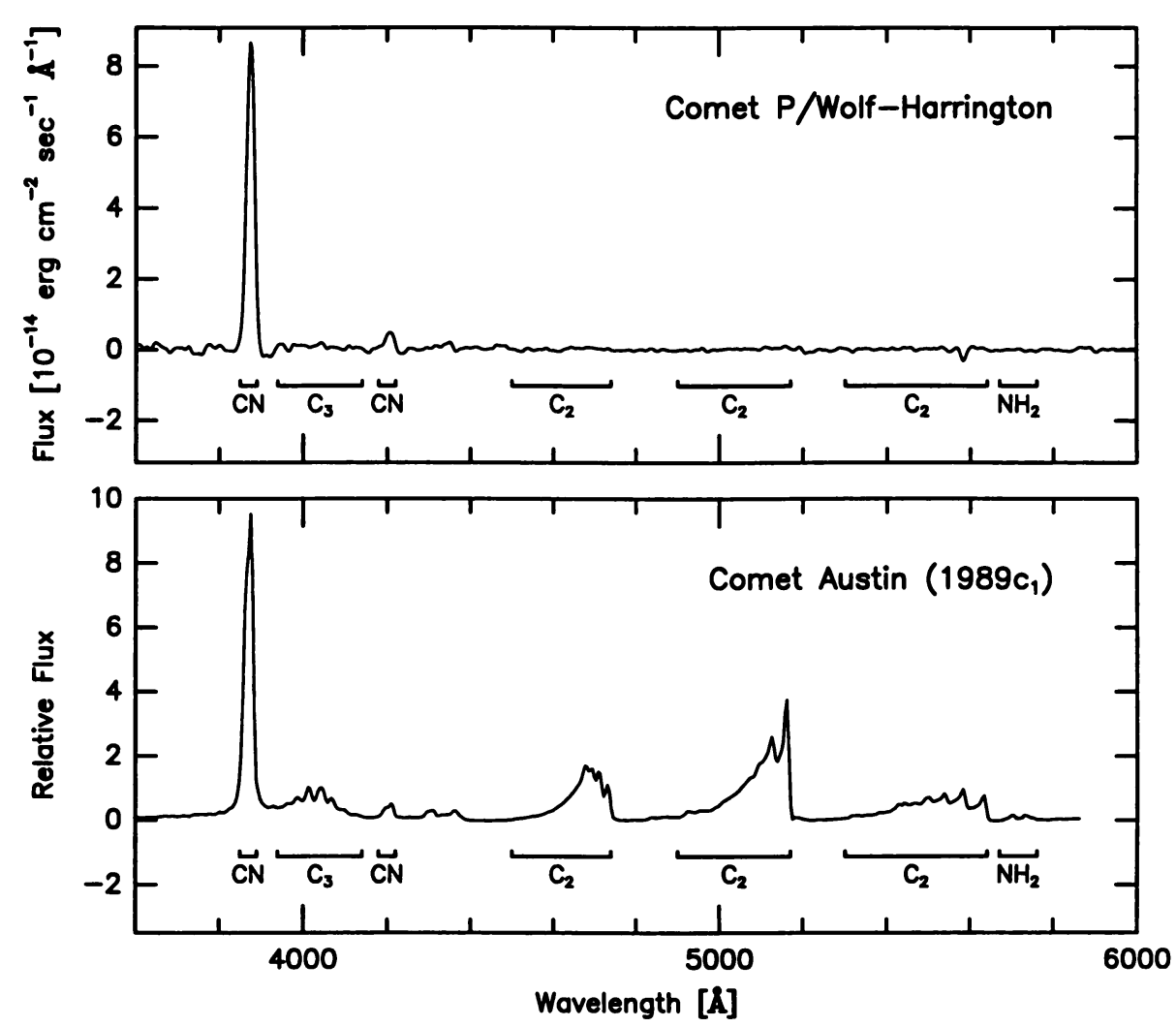

Fig. 5. Spectra of Comets $\mathrm{P} /$ Wolf-Harrington and Austin (1990 V). Note the apparent lack of $C_{2}$ and $C_{3}$ emission in Wolf-Harrington. From Schleicher, Bus, and Osip (1993).

dynamically new comets are often assumed to have a relatively fresh, bright surface as compared to periodic comets which have extremely low albedos. In fact, no albedo measurements have been made of the nucleus of a dynamically new comet, and a variety of processing of the surface is likely to have occurred over 4.5 billion years (cf. Mumma, Weissman, and Stern 1993). These and other lines of evidence for physical evolutionary processes have been reviewed recently by Kresák (1991; 1994) and Meech (1991).

The amount of data available for investigating evolution based on chemical composition is much more limited. Only a small number of comets have had compositional measurements made on multiple apparitions, and a similar small number of dynamically new comets have been observed in the larger database programs discussed in the previous two sections. However, several conclusions have been possible from this limited amount of data. Only 2 of the 11 periodic comets observed at multiple apparitions by A'Hearn et al. (1994) clearly show a change in their water production rate at a given heliocentric distance, and both of these comets 
had undergone significant orbital changes due to perturbations by Jupiter. Moreover, while the total production rates had changed in these comets, the relative abundances of the observed species remained constant. In contrast, variations in abundance ratios are observed to take place in many comets either as a function of heliocentric distance or as an asymmetry about perihelion, but these variations repeat from one apparition to the next. This behavior is interpreted by A'Hearn et al. as evidence for chemical inhomogeneities across the surfaces of some comet nuclei, coupled with changing orientation of the nucleus with respect to the sun. That is to say, variations of this type are believed to be driven by a seasonal effect.

Several compositional trends are observed as functions of orbital parameters, and presumably age or origin. For instance, dynamically new comets apparently preferentially exhibit an excess of pure water ice at large heliocentric distances ( $>3 \mathrm{AU})$ as they arrive near the sun for the first time (cf. A'Hearn et al. 1994). Dynamically new comets also apparently have higher overall production rates before perihelion rather than after, consistent with numerous brightness measurements (A'Hearn et al.). While these results strongly imply that the surface of a dynamically new comet is intrinsically different from the remainder of the material in the nucleus, many more dynamically new comets need to be observed over a large range of distances before these results can be considered conclusive.

A'Hearn et al. also find that while carbon-chain depleted comets constitute approximately $30 \%$ of their taxonomic database, most of these are Jupiter-family comets (i.e., having periods less than 20 years and/or aphelion distances less than $10 \mathrm{AU})$, resulting in about one-half of the observed Jupiter-family comets being classified as carbon depleted. In contrast, most but not all long-period comets are classified as "typical." Since only 1-3 comets from the taxonomic database are dynamically new (two comets have indeterminate values for $1 / a_{0}$ ), the fact that none of these is depleted in the carbon-chain molecules is not statistically significant. A possible scenario consistent with these findings is that the temperature at which the comets formed in the proto-solar nebula not only controlled the basic abundances (cf. Yamamota 1985), but that the critical temperature occurred within the region from which Kuiper Belt comets originated. Therefore, Oort Cloud comets and as many as one-half of Kuiper Belt comets would be classified as "typical," while the remaining Kuiper Belt comets would be in the carbon-chain depleted class (A'Hearn et al. 1994). Alternatively, if about one-half of the Jupiter-family comets actually have an Oort Cloud origin - a fraction significantly greater than is generally assumed - then the A'Hearn et al. taxonomic classes would directly reflect a comet's origin.

A final evolutionary effect is noted for the dust-to-gas ratio observed in the A'Hearn et al. database. While there is no obvious trend with dynamical age, i.e. $1 / a_{0}$, a clear trend with perihelion distance $(q)$ is observed. This correlation is illustrated in Figure 6. A'Hearn et al. note that very little change is seen for the dust-to-gas ratio for a given comet as a function of heliocentric distance, consistent with the findings of Storrs, Cochran, and Barker (1992), ruling out a simple temperature or production rate cause of the correlation with $q$. Since this trend is observed in comets before perihelion as well as after, it also cannot be caused by a simple thermal lag mechanism. A'Hearn et al. postulate that the surface properties 
of the nucleus are controlled by the maximum temperature which it has previously attained, rather than the current temperature, and this in turn controls the release of dust particles observed in the visible. Incorporation of the many results from observations of dust in the IR should test this hypothesis.

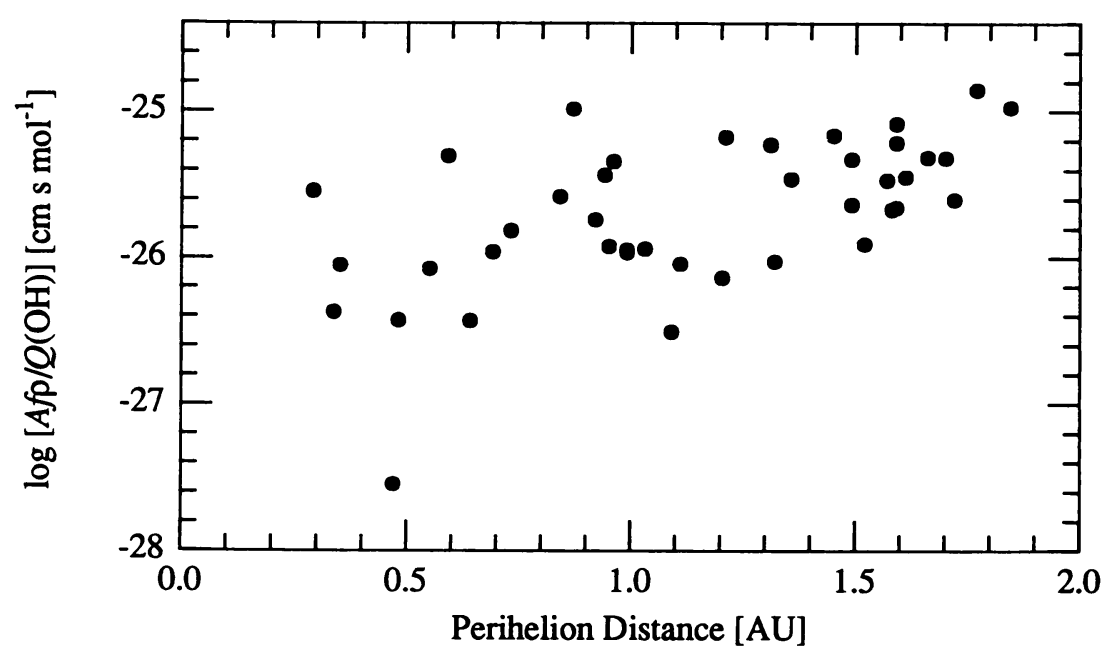

Fig. 6. The dust-to-gas ratio for comets in the A'Hearn et al. restricted database as a function of perihelion distance. Note the clear trend, which may be caused by changes in the surface properties of the cometary nuclei which in turn are controlled by the maximum surface temperature a comet reaches each orbit. From A'Hearn et al. (1994).

\section{Summary}

As evidenced by the many recent results reported on in this paper, the field of comet compositional studies has seen considerable progress in the past decade. Emission by molecular species in the UV and visible portions of the spectrum has now been measured in a sufficient number of comets that taxonomic classifications are not only possible, but have resulted in the discovery of 2 taxonomic groupings by A'Hearn et al. 1994. Moreover, a clear correlation is seen among the three carbonbearing species within the larger, "typical" group, with their respective abundances varying together with respect to water. Evidence that the comets' site of origin is responsible for the basic grouping comes from the comets' orbital parameters, with approximately one-half of the sampled Jupiter-family comets being members of the carbon-chain depleted group, while very few members of this group are Halleyfamily or long-period comets. Correlations of $\mathrm{CN}$ production with dust abundance are observed in many comets, while the amount of dust measured in the visible is observed to vary inversely proportionally with a comet's perihelion distance. This latter effect implies that processing of the surface of a cometary nucleus, rather 
than bulk composition, controls the amount or type of dust grains observed in the cometary coma.

The number of comets observed in the visible will continue to increase in the future, presumably permitting additional taxonomic groupings to be determined. More exciting, however, is the prospect that, due to continued rapid improvements in instrumentation, taxonomic studies should be possible utilizing many additional cometary constituents which are only observed in the IR and microwave regions of the spectrum.

\section{Acknowledgements}

It is a pleasure to thank Mike A'Hearn and Bob Millis for innumerable discussions over many years on a wide range of cometary topics, including the contents of this review. I also thank David Osip for his many contributions, and the referees for their helpful comments. This work was supported by NASA Grant NAGW-1864.

\section{References}

A'Hearn, M. F., and Millis, R. L. : 1980, "Abundance Correlations Among Comets." Astron. J. 85, 1528-1537.

A'Hearn, M. F., Millis, R. L., Schleicher, D. G., Osip, D. J., and Birch, P. V. : 1994, "Narrowband Photometry of Comets : A Database of 85 Comets." In preparation for submittal to Icarus.

Arpigny, C. : 1976, "Interpretation of Comet Spectra." In The Study of Comets (B. Donn et al., Eds.) NASA SP-393, 797-838.

Beaver, J. E., Wagner, R. M., Schleicher, D. G., and Lutz, B. L. : 1990, "Anomalous Molecular Abundances and the Depletion of $\mathrm{NH}_{2}$ in Comet P/Giacobini-Zinner." Astrophys. J. 360, 696-701.

Bobrovnikoff, N. T. : 1927, "On the Spectra of Comets." Astrophys. J. 66, 439-464.

Budzien, S. A., and Feldman, P. D. : 1992, "Upper Limits to the $S_{2}$ Abundance in Several Comets Observed with the International Ultraviolet Explorer." Icarus 99, 143-152.

Cochran, A. L. : 1987, "Another Look at Abundance Correlations Among Comets." Astron. J. 93, 231-238.

Cochran, A. L. : 1990, "Are All Comets Created Equal?" In Asteroids, Comets, and Meteors III (C. Lagerkvist et al., Eds.), 281-284, Uppsala University.

Cochran, A. L., and Barker, E. S. : 1987, "Comet Giacobini-Zinner : A Normal Comet?" Astron. J. 93, 239-243.

Cochran, A. L., Barker, E. S., Ramseyer, T. F., and Storrs, A. D. : 1992, "The McDonald Observatory Faint Comet Survey : Gas Production in 17 Comets." Icarus 98, 151-162.

Cochran, A. L., Green, J. R., Barker, E. S. : 1989, "Are Low-Activity Comets Intrinsically Different from More Active Comets?" Icarus 79, 125-144. Erratum : Icarus 80, 446.

Colom, P., Crovisier, J., Bockellee-Morvan, D., Despois, D., and Paubert, G..: 1992, "Formaldehyde in Comets : I. Microwave Observations of P/Brorsen-Metcalf (1989 X), Austin (1990 V), and Levy (1990 XX)" Astron. Astrophys. 264, 270-281.

Crovisier, J. : 1992, "The Infrared Spectrum of Comets." In Infrared Astronomy with ISO, (T. Encrenaz and M. Kessler, Eds.), 221-248, Nova Science Publishers.

Donn, B. : 1976, "The Nucleus : Panel Discussion." In The Study of Comets (B. Donn et al., Eds.) NASA SP-393, 611-619.

Duncan, M., Quinn, T., and Tremaine, S. : 1988, "The Origin of Short-Period Comets." Astrophys. J. Lett. 328, L69-L73. 
Feldman, P. D. : 1983, "Ultraviolet Spectroscopy and the Composition of Cometary Ice." Science 219, 347-354.

Feldman, P. D. : 1986, "Carbon Monoxide in Cometary Ice." In Asteroids, Comets, and Meteors II (C. Lagerkvist et al., Eds.), 263-267, Uppsala University.

Fink, U. : 1992, "Comet Yanaka (1988r) : A New Class of Carbon-Poor Comet," Science 257, 1926-1929.

Hoban, S., Mumma, M., Reuter, D. C., DiSanti, M., Joyce, R. R., and Storrs, A. : 1991, "A Tentative Identification of Methanol as the Progenitor of the 3.52- $\mu \mathrm{m}$ Emission Feature in Several Comets." Icarus 93, 122-134.

Krankowsky, D. : 1991, "The Composition of Comets." In Comets in the Post-Halley Era (R. Newburn et al., Eds.) vol. 2, 855-877, Kluwer Academic Press.

Kresák, L. : 1991, "Evidence for Physical Aging of Periodic Comets." In Comets in the Post-Halley Era (R. Newburn et al., Eds.) vol. 1, 607-628, Kluwer Academic Press.

Lutz, B. L., Womack, M., and Wagner, R. M. : 1993, "Ion Abundances and Implications for Photochemistry in Comets Halley (1986 III) and Bradfield (1987 XXIX)." Astrophys. J. 407, 402-411.

Meech, K. J. : 1991, "Physical Aging in Comets." In Comets in the Post-Halley Era (R. Newburn et al., Eds.) vol. 1, 629-669, Kluwer Academic Press.

Mumma, M. J., Hoban, S., Reuter, D. C., and DiSanti, M. : 1993, "Methanol in Recent Comets : Evidence for Two Distinct Cometary Populations." In Abstracts for IAU Symposium 160 : Asteroids, Comets, Meteors 1993, LPI Contribution No. 810, 22.

Mumma, M. J., Weissman, P. R, Stern, S. A. : 1993, "Comets and the Origin of the Solar System : Reading the Rosetta Stone." In Protostars and Planets III (E. Levy and J. Lunine, Eds.), 1172-1252, University of Arizona Press.

Newburn, R. L., and Spinrad, H. : 1984, "Spectrophotometry of 17 Comets. I. The Emission Features." Astron. J. 89, 289-309.

Newburn, R. L., and Spinrad, H. : 1989, "Spectrophotometry of 25 Comets : Post-Halley Updates for 17 Comets Plus New Observations for Eight Additional Comets." Astron. J. 97, 552-569.

Rickman, H., Kamél, L., Froeschlé, C., and Festou, M. C. : 1991, "Nongravitional Effects and the Aging of Periodic Comets." Astron. J. 102, 1446-1463.

Schleicher, D. G., Bus, S. J., and Osip, D. J. : 1993, "The Anomalous Molecular Abundances of Comet P/Wolf-Harrington." Icarus 104, 157-166.

Schleicher, D. G., Millis, R. L., and Birch, P. V. : 1987, "Photometric Observations of Comet P/Giacobini-Zinner." Astron. Astrophys. 187, 531-538.

Stern, S. A. : 1990, "ISM-Induced Erosion and Gas-Dynamical Drag in the Oort Cloud." Icarus 84, 447-466.

Storrs, A. D., Cochran, A. L., and Barker, E. S. : 1992, "Spectrophotometry of the Continuum in 18 Comets." Icarus 98, 163-178.

Tholen, D. J. : 1984, "Asteroid Taxonomy from Cluster Analysis of Photometry." Ph. D. Dissertation, University of Arizona.

Weaver, H. A., Feldman, P. D., Festou, M. C., A'Hearn, M. F., and Keller, H. U. : 1981, "IUE Observations of Faint Comets." Icarus 47, 449-463.

Whipple, F. L. : 1977, "The Constitution of Cometary Nuclei." In Comets, Asteroids, Meteorites (A. Delsemme, ed.), 25-34, Univ. of Toledo Press.

Yamamoto, T.: 1985, "Formation History and Environment of Cometary Nuclei." In Ices in the Solar System (J. Klinger et al., Eds.), 205-219, D. Reidel Publishing Co. 